\title{
Retrospective Study of the Significant Predictive Role of Inflammatory Degree in Initial and Repeat Prostate Biopsy Specimens for Detecting Prostate Cancer
}

\author{
Sung Han Kim, MD, PhD \\ Boram Park, MS² \\ Jae Young Joung, MD, $\mathrm{PhD}{ }^{1}$ \\ Jinsoo Chung, MD, PhD' \\ Ho Kyung Seo, MD, PhD' \\ Kang Hyun Lee, MD, PhD' \\ Weon Seo Park, MD, PhD
}

${ }^{1}$ Department of Urology, Center for Prostate Cancer, National Cancer Center, Goyang, ${ }^{2}$ Biostatistics Collaboration Unit, Research Institute, National Cancer Center, Goyang, ${ }^{3}$ Department of Pathology, Center for Prostate Cancer, National Cancer Center. Goyang, Korea
Correspondence: Weon Seo Park, MD, PhD Department of Pathology and Center for Prostate Cancer, Research Institute and Hospital of National Cancer Center,

323 Ilsan-ro, Ilsandong-gu, Goyang 10408, Korea Tel: 82-31-920-1744

Fax: 82-31-920-1369

E-mail: thymus@ncc.re.kr

Co-correspondence: Kang Hyun Lee, MD, PhD Department of Urology and Center for Prostate Cancer, Research Institute and Hospital of

National Cancer Center, 323 Ilsan-ro,

Ilsandong-gu, Goyang 10408, Korea

Tel: 82-31-920-2799

Fax: 82-31-920-1676

E-mail: uroonco@ncc.re.kr

Received May 29, 2018

Accepted October 1, 2018

Published Online October 2, 2018

\section{Purpose}

The purpose of this study was to determine whether histologic inflammation (HI) in initial and repeat prostate biopsy specimens was significantly associated with the detection of prostate cancer.

\section{Materials and Methods}

Between 2005 and 2017, the clinicopathological records of patients with high prostatespecific antigen (PSA) levels who underwent initial and repeat prostate biopsies were retrospectively reviewed. The presence of $\mathrm{HI}$ and its degree in each biopsied specimen were interpreted by one uropathologist with 20 years of experience. The association between $\mathrm{HI}$ and cancer diagnosis was statistically assessed, with $p<0.05$ considered significant, and the cancer and non-cancer groups were compared.

\section{Results}

Among the 522 patients with a median PSA levels of $6.5 \mathrm{ng} / \mathrm{dL}$, including $258(49.4 \%)$ whose cancer was diagnosed following repeat biopsy, the median degrees of $\mathrm{HI}$ in the initial and repeat biopsies were $25.0 \%$ and $41.7 \%$, respectively. Furthermore, 211 (40.4\%) and 247 (47.3\%) patients had $\mathrm{HI}$ (> 0\%) on biopsied specimens, respectively. Comparison of the cancer and noncancer groups revealed that a greater rate of $\mathrm{HI}$ specimens in the initial biopsy was associated with fewer prostate cancer diagnoses following repeat biopsy ( $p<$ 0.001). Other comparisons between the cancer and non-cancer groups showed that the cancer group had a significantly higher rate of hypertension, whereas those non-cancer group had a significantly higher rate of benign prostatic hyperplasia and prostatitis ( $p<$ 0.05).

\section{Conclusion}

A finding of a lesser degree of $\mathrm{HI}$ in the initial and a greater degree of $\mathrm{HI}$ in the repeat biopsied specimens was associated with the higher probability of cancer diagnosis in patients with high PSA levels.
Key words Prostate, Biopsy, Prostate-specific antigen, Inflammation, Neoplasms 


\section{Introduction}

Since the discovery of prostate-specific marker, namely, prostate-specific antigen (PSA), the PSA level is one of the screening markers for prostate biopsies, along with digital rectal examination (DRE) and transrectal ultrasonography (TRUS) [1]. PSA levels $>3.0 \mathrm{ng} / \mathrm{mL}$ are correlated with prostate cancer (PC); however, PSA is not totally PC specific, with a wide range of sensitivity, that is, between $21 \%$ and $95 \%$. It varies with prostate volume, histologic inflammation (HI), urethral instrumentation, and amount of cancer within the gland to exhibit limited diagnostic power for cancer detection. Several supplementary PSA parameters such as PSA velocity (change of PSA over time), PSA density (standardization of PSA level in relation to the size of the prostate), adjusted PSA reference ranges (accounting for age-dependent prostate growth and occult prostatic disease), and PSA forms have been developed to maximize the accuracy of PC predictability [1].

Patients with a high suspicion of PC and concurrent elevated PSA level, as well as abnormal findings on DRE or TRUS, were recommended to undergo a TRUS-guided prostate biopsy (TRUS-Bx) [1-3]. Because of the invasiveness of TRUS-Bx and its related complications, the dilemma of recommending TRUS-Bx for patients with a high PSA level has gained attention. Another issue is the difficulty for clinicians in assuring patients who are not worried about PC because of the possible predisposition to PC with false-negative results from TRUS-Bx; these patients typically yield a negative cancer pathology with some HI. Further, these patients are generally recommended to undergo a repeat TRUS-Bx, despite having received anti-inflammatory treatment [2].

For urologists, it is important to find predisposing factors to eliminate PSA level elevation due to $\mathrm{HI}$ and to avoid unnecessary repeat TRUS-Bx. Previous literature has suggested a prevalence range of 22\%-63\% for acute $\mathrm{HI}$ and $8 \%-99 \%$ for chronic HI among asymptomatic men who were biopsied due to an elevated PSA level [4]. This elucidates the strong relationship between chronic $\mathrm{HI}$ and PC risk, which suggests a protective effect of anti-inflammatory agents against PC $[5,6]$. HI associated with proliferative inflammatory atrophy has also been proposed as a precursor for prostatic intraepithelial neoplasia and PC [7].

Therefore, in this study, the degree of HI in initial and repeat biopsied prostate specimens was evaluated to determine whether it was correlated with PC in patients with a high suspicion PC who were diagnosed as PC-negative on the initial biopsy, but were diagnosed with $\mathrm{PC}$ on the repeat biopsy.

\section{Materials and Methods}

\section{Patients and tissue samples}

Study participants included patients with high PSA levels who underwent the initial TRUS-Bx either at an outside hospital $(n=424,81.2 \%)$ or the Prostate Cancer Center, National Cancer Center in Goyang, Korea $(\mathrm{n}=98,18.8 \%)$, and the repeat TRUS-Bx at the National Cancer Center (all participants), between 2005 and 2017. All the patients included in this study had at least 12 core biopsies. Those patients who had prostatic intraepithelial neoplasia, high grade, atypical adenomatous hyperplasia, and atypical small acinar proliferation at initial biopsy were all excluded. And those patients who did not undergo the systemic 12 biopsies or had only several targeted biopsies or did not have information about the initial outside biopsy were all excluded. Their clinicopathological records including underlying disease, initial laboratory blood tests with PSA levels before and after biopsy, Gleason score, and clinical TN stages for those diagnosed PC on repeat biopsy were reviewed. All patients had only two pathological reports of initial and repeat prostate biopsies without subsequent later biopsies. The prostatic biopsied indications were high PSA of $>3.0 \mathrm{ng} / \mathrm{dL}$, at least three 3-month-interval consecutive PSA rise despite antibiotic or anti-inflammatory management, prostatic nodule on DRE, and abnormal low-echoic lesion suggesting suspicious malignancy on TRUS.

The TRUS-Bx under local anaesthesia was performed by a single uroradiologist with 20 years of experience. Systemic twelve TRUS-Bx were routinely performed with or without one or two additional targeted-core biopsies, if necessary. The repeat biopsy after the initial biopsy without cancer diagnosis was indicated to be performed within 4-8 weeks after the initial biopsy with sufficient anti-inflammatory treatment or $>2$ weeks of antibiotics treatments according to the urologists' discretion when the PSA level continued to rise or remained similar to the initial PSA levels with/without abnormal nodular lesions or hypoechoic lesions either on DRE or TRUS.

Each 12 biopsy cores with/without one or two additional cores were fixed in buffered formalin and embedded into paraffin blocks and stained with haematoxylin and eosin. The total biopsy length was calculated by adding biopsy core lengths together. The presence of $\mathrm{HI}$ and its degree in each biopsied specimen were routinely interpreted on the basis of the presence of mononuclear or polymorphonuclear leukocyte infiltration by one uropathologist (W.S.P.) with 20 years of experience, who was blinded to the study's aims concerning the original diagnosis of the initial biopsy and the subsequent biopsy (Fig. 1). As for the 438 (83.9\%) referred patients 


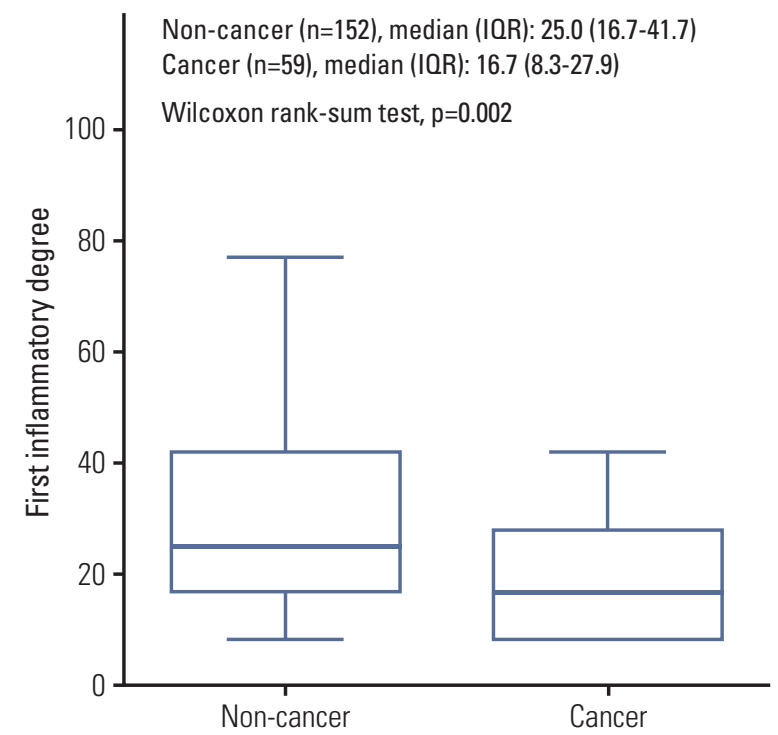

A

Fig. 1. The different degree (\%) of prostatic inflammation in initial (A) and repeat (B) biopsied specimens between noncancer and cancer groups in $>0 \%$ inflammatory degree patients.
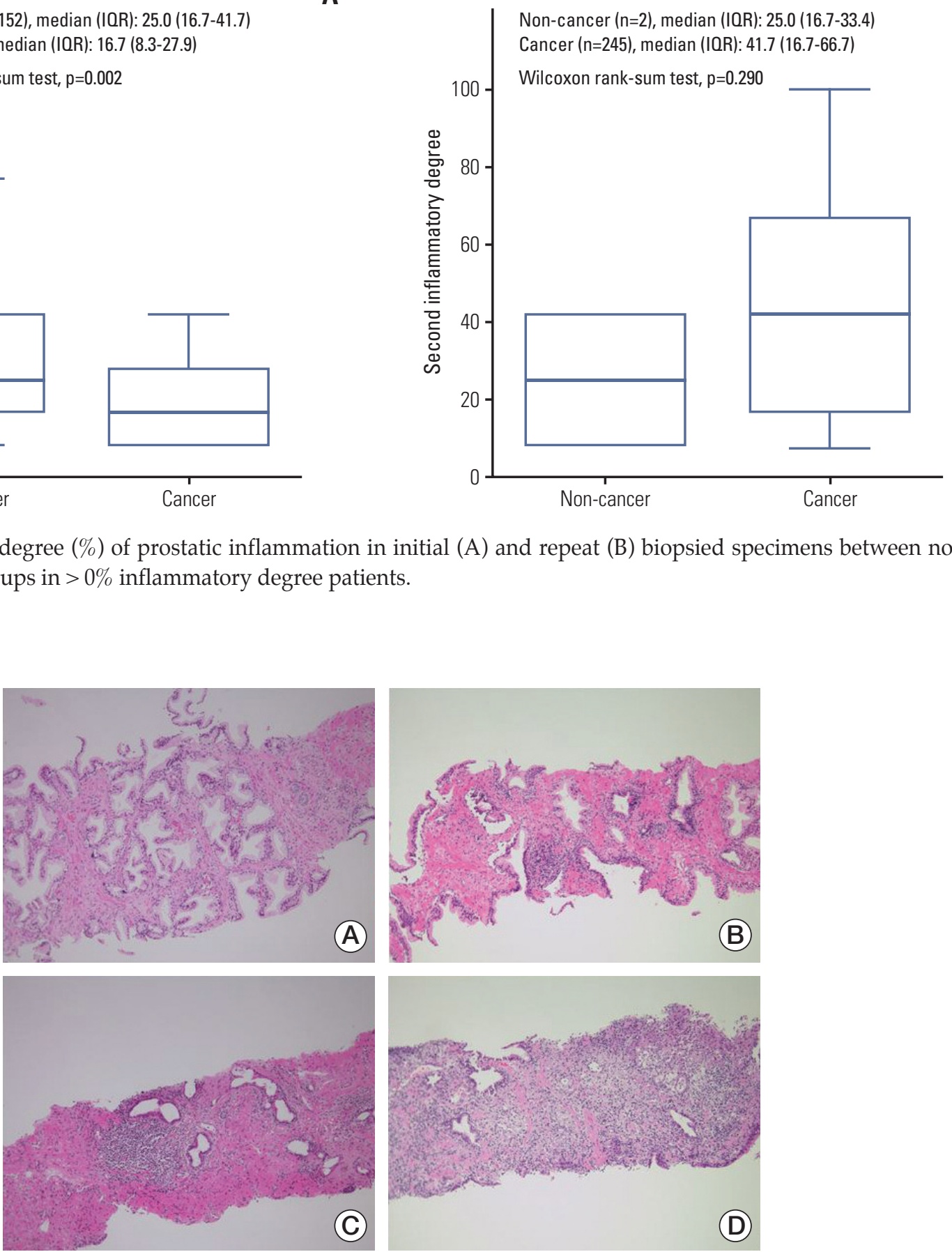

Fig. 2. Inflammation degree at prostate biopsied specimens. (A) No inflammation. (B) Mild inflammation. (C) Moderate inflammation. (D) Severe inflammation.

after initial biopsy from other hospitals, all the 12 (+2 additional targeted biopsy) systemic biopsied slides were brought to be reviewed unblended manner by the uropathologist to define the degree of HI (Fig. 2). Any slide detected with inflammatory cells was positive under microscopy and the percentage degree (0\%-100\%) was expressed with the positive inflammatory slides divided by the total number of slides (between 12 and 14 biopsied slides). The degree of HI 
was recorded routinely in the pathology report as continuous $(0 \%-100 \%)$ and binary (0\% vs. $1 \%-100 \%)$ variables.

\section{Statistical analysis}

The association between $\mathrm{HI}$ and cancer diagnosis and the comparison between the cancer and non-cancer groups were statistically analysed using Student's t test, Wilcoxon's ranksum test, Pearson's chi-square test, and the binary logistic regression model with, $\mathrm{p}<0.05$ considered significant. The sensitivity, specificity, positive predictive value (PPV), and negative predictive value (NPV) of the HI degree in the inital biopsied specimens were assessed to determine the adequate predictive value of $\mathrm{HI}$ degree. All analyses were performed by a biostatistician (B.P.) using SAS software, ver. 9.4 (SAS Institute Inc., Cary, NC), and R software, ver. 3.3.3 (R Project for Statistical Computing, Vienna, Austria).

\section{Ethical statement}

All study protocols were conducted according to the ethical guidelines of the "World Medical Association Declaration of Helsinki-Ethical Principles for Medical Research Involving Human Subjects." This study was approved by the Institutional Review Board of the Research Institute and Hospital National Cancer Center (IRB No. NCC2018-0012). Written informed consent was waived from the IRB.

Table 1. Summary of clinico-pathologic characteristics

\begin{tabular}{|c|c|c|c|c|}
\hline & \multirow{2}{*}{ Total $(n=522)$} & \multicolumn{2}{|c|}{ Cancer } & \multirow{2}{*}{ p-value } \\
\hline & & No $(n=264)$ & Yes $(n=258)$ & \\
\hline Age (yr) & $67.2 \pm 8.7$ & $65.1 \pm 7.8$ & $69.3 \pm 9.0$ & $<0.001$ \\
\hline Body mass index $\left(\mathrm{kg} / \mathrm{m}^{2}\right)$ & $24.1 \pm 3.0$ & $24.3 \pm 3.0$ & $24.0 \pm 2.9$ & 0.282 \\
\hline PSA (ng/dL) & $6.5(4.3-12.6)$ & $5.2(3.7-8.2)$ & $9.9(5.4-28.8)$ & $<0.001$ \\
\hline \multicolumn{5}{|l|}{ Underlying disease } \\
\hline Benign prostatic hyperplasia & 337 (64.6) & $211(79.9)$ & $126(48.8)$ & $<0.001$ \\
\hline Prostatitis & $44(8.4)$ & $34(12.9)$ & $10(3.9)$ & $<0.001$ \\
\hline Diabetes & $78(14.9)$ & $38(14.4)$ & $40(15.5)$ & 0.722 \\
\hline Hypertension & $219(42.0)$ & $94(35.6)$ & $125(48.5)$ & 0.003 \\
\hline Cardio disease & $30(5.8)$ & $13(4.9)$ & $17(6.6)$ & 0.414 \\
\hline Brain disease & $17(3.3)$ & $9(3.4)$ & $8(3.1)$ & 0.843 \\
\hline \multicolumn{5}{|l|}{ Pre-biopsied blood analysis } \\
\hline Leukocyte & $6.2(5.2-7.2)$ & $6.2(5.2-7.3)$ & $6.2(5.2-7.2)$ & 0.934 \\
\hline Lymphocyte & $31.7(25.8-38.4)$ & $32.9(26.6-38.6)$ & $30.6(25.4-38.3)$ & 0.261 \\
\hline Monocyte & $7.5(6.3-9.0)$ & $7.1(6.2-8.8)$ & $7.6(6.3-9.3)$ & 0.164 \\
\hline Neutrophil & $56.2(50.3-63.2)$ & $55.2(50.0-62.6)$ & $56.7(51.0-63.9)$ & 0.258 \\
\hline Platelet & $221(186-259)$ & $223.5(181.8-266.2)$ & $219.0(188.2-250.5)$ & 0.427 \\
\hline Absolute neutrophil count & $3,390(2,633-4,312)$ & $3,342(2,582-4,300)$ & $3,459(2,749-4,310)$ & 0.595 \\
\hline Alkaline phosphatase & $68.5(58.0-85.0)$ & $69.0(56.3-82.8)$ & $68.0(58.3-86.0)$ & 0.363 \\
\hline Creatinine & $0.9(0.8-1.1)$ & $0.9(0.8-1.0)$ & $0.9(0.8-1.1)$ & 0.993 \\
\hline Blood urea nitrogen & $15(13-19)$ & $15(13-18)$ & $16(13-19)$ & 0.129 \\
\hline Transaminases aspartate transaminase & $22(18-28)$ & $21(18-26)$ & $23(19-29)$ & 0.067 \\
\hline Alanine transaminase & $20(15-28)$ & $21(15-27)$ & $20(14-28)$ & 0.400 \\
\hline \multicolumn{5}{|l|}{ Initial biopsied inflammatory degree $(\%)$} \\
\hline 0 & $311(59.6)$ & $112(42.4)$ & $199(77.1)$ & $<0.001$ \\
\hline$>0$ & $211(40.4)$ & $152(57.6)$ & $59(22.9)$ & \\
\hline Median (IQR) & $25.0(16.7-33.3)$ & $25.0(16.7-41.7)$ & $16.7(8.3-27.9)$ & 0.002 \\
\hline \multicolumn{5}{|l|}{ Repeat biopsied inflammatory degree $(\%)$} \\
\hline 0 & $275(52.7)$ & $262(99.2)$ & $13(5.0)$ & $<0.001$ \\
\hline$>0$ & $247(47.3)$ & $2(0.8)$ & $245(95.0)$ & \\
\hline Median (IQR) & $41.7(16.7-66.7)$ & $25.0(16.7-33.4)$ & $41.7(16.7-66.7)$ & 0.290 \\
\hline
\end{tabular}

Values are presented mean \pm standard deviation, median (interquartile range), or number $(\%)$. 


\section{Results}

\section{Baseline characteristics}

Among the 522 enrolled patients, 258 patients (49.4\%) were diagnosed with PC on repeat biopsy. The mean age and median initial PSA level were 67.2 years and $6.5 \mathrm{ng} / \mathrm{dL}$ respectively. On the initial prostate biopsied specimens, 211 patients $(40.4 \%)$ had $\mathrm{HI}$ with a median inflammatory extent of $25 \%(16.7 \%-33.3 \%)$. On repeat prostate biopsied specimens, 247 patients $(47.3 \%)$ had a median inflammatory extent of $41.7 \%(16.7 \%-66.7 \%)$ (Table 1$)$.

\section{The baseline comparison between the cancer and non- cancer groups}

The comparison of baseline characteristics including laboratory parameters and underlying diseases showed that the cancer group $(n=258)$ had significantly older, higher initial PSA level and lesser proportion of benign prostatic hyperplasia and prostatitis with a higher rate of hypertension than the non-cancer group $(\mathrm{n}=264, \mathrm{p}<0.05)$ (Table 1). Comparison of the cancer revealed that a greater $\mathrm{HI}$ specimen in the initial biopsy was associated with fewer PC diagnoses following repeat biopsy $(\mathrm{p}<0.001)$. The rate of patients with $\mathrm{HI}$ on initial $(57.6 \%$ vs. $22.9 \%)$ and repeat biopsied (0.8\% vs. $95.0 \%)$ specimens also showed significant differences $(\mathrm{p}<0.001)$. A significantly lesser degree of $\mathrm{HI}$ on initial biopsy (a median degree of $\mathrm{HI}, 25.0 \%$ vs. $16.7 \%$; $\mathrm{p}=0.002$ ) and insignificantly higher degree of $\mathrm{HI}$ on repeat biopsy (25.0\% vs. $41.7 \%$, $\mathrm{p}=0.290$ ) were detected in the cancer group (Table 1). Among the cancer group, the pathological characteristics showed that a median of $33.3 \%$ of tumour was detected on repeat biopsy with Gleason scores $8(11.1 \%)$ and $9(13.5 \%)$. The clinical TN stages were $20.4 \%, 11.0 \%$, and $19.2 \%$ of cT3, cT4, and cTxN1, respectively (Table 2).

\section{Logistic regression model for cancer diagnosis in initial and repeat biopsy}

The degree of HI on initial biopsy had significant association with PC in univariable and multivariable models ( $\mathrm{p}<$ 0.001) (Table 3). In univariable model, the patients with HI had lower PC diagnoses than the patients without HI (odds ratio [OR], $0.22 ; 95 \%$ confidence interval [CI], 0.15 to $0.32 ; \mathrm{p}$ $<0.001)$. The similar results were obtained in the multivariable model with adjusted age and PSA (OR, 0.22; 95\% CI, 0.15 to $0.33 ; \mathrm{p}<0.001 ; \mathrm{OR}, 0.30 ; 95 \% \mathrm{CI}, 0.20$ to $0.44 ; \mathrm{p}<0.001$, for each model). Diagnostic tests to determine the sensitivity, specificity, PPV, and NPV were performed at various cut-off points of HI degree in the initial biopsy (S1 Table). If PC was
Table 2. The cancer-diagnosed patients' characteristics

\begin{tabular}{|cc}
\hline Tumour volume percentage $\mathbf{( 0 \% - 1 0 0 \% )}$ & Value (n=258) \\
Apex tumour & $33.3(16.7-58.3)$ \\
No & $54(21.4)$ \\
Yes & $198(78.6)$ \\
Gleason score & \\
\hline 6 & $107(42.5)$ \\
\hline 7 & $83(32.9)$ \\
\hline 8 & $28(11.1)$ \\
\hline 9 & $34(13.5)$ \\
\hline Clinical T category & \\
\hline T1 & $89(36.3)$ \\
T2 & $79(32.2)$ \\
\hline T3 & $50(20.4)$ \\
\hline T4 & $27(11.0)$ \\
\hline Clinical N category & \\
\hline N0 & $198(80.8)$ \\
\hline N1 & $47(19.2)$ \\
\hline Clinical stage grouping & \\
\hline cT1c-cT2b & $135(55.1)$ \\
\hline cT2c & $28(11.4)$ \\
\hline cT3-cT4 & $35(14.3)$ \\
\hline LN+ & $47(19.2)$ \\
\hline
\end{tabular}

Values are presented as median (interquartile range) or number $(\%)$.

predicted based on $0 \% \mathrm{HI}$ degree, the performance values were $77.1 \%$ and $57.6 \%$ of sensitivity and specificity, respectively. Additionally, if PC was predicted based on HI degree with one of the 12 biopsy cores $(8.3 \%)$, the performance was $84.1 \%$ of sensitivity and $46.6 \%$ of specificity.

\section{The subgroup analysis for patients underwent both ini- tial and repeat biopsy at same institution}

As for the 98 patients who underwent initial and repeat TRUS-Bxs at the National Cancer Center, the comparison of baseline characteristics between the cancer and non-cancer groups showed similar statistical results as that of all 522 patients, with the exception of prostatitis and hypertension being the insignificantly different underlying disease $(\mathrm{p}<$ 0.05). Additionally, the degree of $\mathrm{HI}$ of the initial biopsied specimen (analysed as a continuous variable) was not significantly different between the cancer and non-cancer groups ( $p=0.291$ ) (Table 4). Diagnostic tests for the sensitivity, specificity, PPV, and NPV among the 98 patients showed that if PC was predicted based on a $0 \% \mathrm{HI}$ degree, the performance values were $71.1 \%$ and $55.0 \%$ for sensitivity and specificity, respectively. Additionally, if PC was predicted based on the 
Table 3. Univariable and multivariable model logistic regression model for cancer diagnosis in initial and repeat biopsy

\begin{tabular}{|c|c|c|}
\hline & Odds ratio $(95 \% \mathrm{CI})$ & p-value \\
\hline Age & $1.06(1.04-1.08)$ & $<0.001$ \\
\hline Body mass index & $0.97(0.91-1.03)$ & 0.281 \\
\hline PSA & $1.06(1.03-1.08)$ & $<0.001$ \\
\hline \multicolumn{3}{|l|}{ Initial biopsied inflammatory degree } \\
\hline \multicolumn{3}{|l|}{ Crude model } \\
\hline No & 1 (reference) & \\
\hline Yes & $0.22(0.15-0.32)$ & $<0.001$ \\
\hline Amount $(\%)$ & $0.97(0.95-0.99)$ & 0.003 \\
\hline \multicolumn{3}{|l|}{ Adjusted for age } \\
\hline No & 1 (reference) & \\
\hline Yes & $0.22(0.15-0.33)$ & $<0.001$ \\
\hline Amount $(\%)$ & $0.97(0.95-0.99)$ & 0.002 \\
\hline \multicolumn{3}{|l|}{ Adjusted for age, PSA } \\
\hline No & 1 (reference) & \\
\hline Yes & $0.30(0.20-0.44)$ & $<0.001$ \\
\hline Amount $(\%)$ & $0.97(0.95-0.99)$ & 0.002 \\
\hline \multicolumn{3}{|l|}{ Repeat biopsied inflammatory degree } \\
\hline Crude model; amount (\%) & $1.04(0.96-1.12)$ & 0.356 \\
\hline Adjusted for age; amount ( $\%)$ & $1.04(0.96-1.12)$ & 0.361 \\
\hline Adjusted for age, PSA; amount (\%) & $1.04(0.96-1.12)$ & 0.359 \\
\hline
\end{tabular}

CI, confidence interval; PSA, prostatic specific antigen.

Table 4. Summary of clinicopathologic characteristics

\begin{tabular}{|c|c|c|c|c|}
\hline & \multirow{2}{*}{ Total $(\mathrm{n}=98)$} & \multicolumn{2}{|c|}{ Cancer } & \multirow{2}{*}{ p-value } \\
\hline & & No $(n=60)$ & Yes $(n=38)$ & \\
\hline Age (yr) & $68.1 \pm 7.8$ & $66.3 \pm 6.2$ & $71.1 \pm 9.1$ & 0.005 \\
\hline Body mass index $\left(\mathrm{kg} / \mathrm{m}^{2}\right)$ & $24.3 \pm 2.9$ & $24.8 \pm 3$ & $23.6 \pm 2.7$ & 0.052 \\
\hline PSA (ng/dL) & $6.8(3.8-10.6)$ & $6.3(3.7-9.8)$ & $9.1(3.8-11.5)$ & 0.221 \\
\hline \multicolumn{5}{|l|}{ Underlying disease } \\
\hline Benign prostatic hyperplasia & $70(71.4)$ & $54(90.0)$ & $16(42.1)$ & $<0.001$ \\
\hline Prostatitis & $5(5.1)$ & $5(8.3)$ & 0 & 0.153 \\
\hline Diabetes & $11(11.2)$ & $6(10.0)$ & $5(13.2)$ & 0.746 \\
\hline Hypertension & $38(38.8)$ & $24(40.0)$ & $14(36.8)$ & 0.755 \\
\hline Cardio disease & $3(3.1)$ & $2(3.3)$ & $1(2.6)$ & $>0.999$ \\
\hline Brain disease & $2(2.0)$ & $2(3.3)$ & 0 & 0.520 \\
\hline \multicolumn{5}{|c|}{ Initial biopsied inflammatory degree $(\%)$} \\
\hline 0 & $54(55.1)$ & $27(45.0)$ & $27(71.1)$ & 0.012 \\
\hline$>0$ & $44(44.9)$ & $33(55.0)$ & $11(29.0)$ & \\
\hline Median (IQR) & $33.3(16.7-41.7)$ & $33.3(16.7-50.0)$ & $25(16.7-41.7)$ & 0.291 \\
\hline \multicolumn{5}{|c|}{ Repeat biopsied inflammatory degree $(\%)$} \\
\hline 0 & $63(64.3)$ & $59(98.3)$ & $4(10.5)$ & $<0.001$ \\
\hline$>0$ & $35(35.7)$ & $1(1.7)$ & $34(89.5)$ & \\
\hline Median (IQR) & $33.3(16.7-50.0)$ & $8.3(8.3-8.3)$ & $33.3(16.7-50.0)$ & 0.148 \\
\hline
\end{tabular}

Values are presented mean \pm standard deviation, median (IQR), or number (\%). PSA, prostatic specific antigen; IQR, interquartile range. 
degree of $\mathrm{HI}$ from one of the 12 biopsy cores $(8.3 \%)$, the performance was $73.7 \%$ for sensitivity and $48.3 \%$ for specificity (S2 Table).

\section{Discussion}

Despite the initial prostate biopsy yielding negative results for PC, a repeat prostate biopsy is indicated for differentiating PC from other benign prostatic diseases in high-risk patients during active surveillance. This study focused on both the initial and repeat biopsies in relation to $\mathrm{HI}$ in the biopsied prostate specimens for screening of $\mathrm{PC}$ in patients with a high suspicion of PC.

$\mathrm{HI}$ and tumorigenesis are known to be closely related. Among the diverse aetiologies of PC, several instances of chronic $\mathrm{HI}$ can cause tumours, and the interaction between immune cells can stimulate tumour cell proliferation, progression, and angiogenesis through producing reactive oxygen species and inflammatory processes, resulting in protein and tissue damage and permanent DNA damage [8,9]. Activation of oncogenes, inactivation of anti-oncogenes or chromosomal rearrangements, and other reasons may also be related to inflammatory processes that activate the nuclear transcription factor in tumour cells, which then promote the tumour cells to produce more chemokines, cytokines, cyclooxygenase 2 , and vascular endothelial growth factor, as well as other inflammatory factors [10-12].

$\mathrm{HI}$ is not only one of the risk factors of $\mathrm{PC}$, it is also associated with proliferative inflammatory atrophy and prostatic intraepithelial neoplasia, which are precursors of PC cell development $[5,13]$. The rate of PC incidence reduces after controlling $\mathrm{HI}$ with anti-inflammatory agents, which further supports the close relationship between HI and PC development $[4,14]$. A recent study showed that mRNA and protein expression levels of inflammatory factors, such as inducible nitric oxide synthase, vascular endothelial growth factor, interleukin-2 and -8 , and tumour necrosis factor $\alpha$, were significantly increased in PC, as well as in the benign prostatic hyperplasia+prostatitis group compared to the benign prostatic hyperplasia group $(p<0.05)$ [11]. Vasavada et al. [14] performed a meta-analysis including 20,585 subjects and 6,641 PC cases and demonstrated that HI on TRUS-Bx was associated with lower PC risk, which was similar to the findings of this study.

However, other biopsy studies, one recent meta-analysis, and another retrospective immunohistochemical study reported no significant association or borderline negative association between $\mathrm{HI}$ on biopsied prostate specimens and PC screening on TRUS-Bx [6]. Yli-Hemminki et al. [4] used the histological grading (0-3) degree of acute and chronic $\mathrm{HI}$ states in 293 initial prostate biopsies, and indicated that HI in a prostate biopsy was not a useful risk indicator of PC screening.

The present study also showed the significant association between HI on biopsied specimens and PC. Specifically, a significantly lower degree of $\mathrm{HI}$ on initial biopsy and an insignificantly greater degree of $\mathrm{HI}$ on repeat biopsy were related to PC diagnosis $(\mathrm{p}<0.05)$ (Table 3 ). This significant finding of HI on initial TRUS-Bx was similar to that of Vasavada et al. [14], and the insignificant HI on repeat TRUS-Bx was similar to the findings presented by Yli-Hemminki et al. [4]. This indicates that already known prostatitis or BPH with rapidly increasing PSA level within a short interval was likely caused by HI, and not by PC itself. The complete absence of $\mathrm{HI}$ or at least one core of positive $\mathrm{HI}$ on initial biopsy in high-risk patients had a sensitivity between $77.1 \%$ $84.1 \%$, with a NPV of $72 \%-75 \%$ and a specificity of cancer screening of $46.6 \%-57.6 \%$ (S1 and S2 Tables).

The insignificantly greater $\mathrm{HI}$ on repeat biopsy relating to PC was further analysed in response to the time interval between the initial and repeat biopsies. In the biopsy slides of the 98 patients who underwent both initial and repeat biopsies at the National Cancer Center, PC was detected with greater $\mathrm{HI}$ on the repeat biopsy with a shorter time interval between biopsies. This meant that the abruptly increased HI on the prostate with increased PSA velocity had a higher chance of having PC, whereas those with slowly increasing HI with slow PSA velocity had a lesser degree of PC (data not shown) [15].

Thereby, several alternative strategies exist for identifying men who may be at risk for PC, despite negative findings on biopsy sample analysis [2]. The use of other serum PSArelated and urinary measurements, such as urinary transmembrane protease serine 2-E26 transformation specific family membrane gene fusion RNA, enable more sensitive and specific diagnosis and can be combined with the knowledge of clinicopathological features to improve outcomes. Other options include the Food and Drug Administration-approved Progensa PCA3 RNA test and prostate imaging using magnetic resonance imaging (MRI). Newer tissue-based assays that measure methylation changes in normal prostate tissue are currently being developed. A cost-effective strategy is proposed in order to address this challenging clinical scenario, and potential directions of future studies in this area are also described.

As for the clinically significant $\mathrm{PC}$, about $57.5 \%$ of patients had a significant Gleason score of $>7$, and $33.5 \%$ had advanced PC (T3-4N0M0, LN+) (Table 2). Most of the PC had apical lesion tumours $(78.6 \%)$, similar to previous studies [16]. Prostate biopsy via the transrectal approach has some limitations with regards to underdetection and undergrading 
of clinically significant PC and the number of cores and location for systematic sampling due to the fact that the direction of the ultrasonographic probe is not easily accessible to the apex of the prostate, as well as the risk of bleeding at the hypervascular apical area $[16,17]$. Concurrent multiparametric MRI with transperineal prostatic biopsy was recently proposed as a solution to reduce overdiagnosis and subsequent overtreatment, with a $44 \%-87 \%$ detection rate of significant PC; this was similar to the $49.4 \%$ missed diagnosis rate from initial biopsy in this study $[18,19]$. These findings are notable as other studies have reported a 10\%-22\% detection rate on repeat biopsy [20]. However, a limitation exists for the multiparametric MRI, especially for detecting apical tumours. Lesions that are small in size and are located at the margin of the prostate are unnoticed on diffusion-weighted imaging protocols due to signals received from surrounding benign prostatic tissue that overshadows the lesion [21,22]. In response, Ukimura et al. [17] advised to obtain samples from the anterior apex, anterior lateral horn, and anterior transition zone in the repeat TRUS-Bx to increase the accuracy of detecting PC [23].

This study had several limitations, including its retrospective design and small-sized biopsied samples. Further imaging characteristics of MRI in the cancer group were not assessed to correlate with the HI degree of histologic slides for better understanding of the $\mathrm{HI}$ aspects on PC, and the insignificant inflammatory laboratory parameters were not discussed. However, this was the first study to evaluate the extent of HI degree in percentages in both initial and repeat biopsied prostate specimens, and the current debate and clinical dilemma of the significant role of $\mathrm{HI}$ of prostate biopsy have been proven in concordance with previous studies. Further studies with prospectively designed biopsies are warranted to solve the serum inflammatory parameters including cytokines and the degree of inflammation on prostate for those PC and non-PC patients.

This study showed a significantly close association between prostatic inflammation and PC on both initial and repeat biopsies. A finding of a lesser degree of $\mathrm{HI}$ in the initial and a greater degree of $\mathrm{HI}$ in the repeat biopsied specimens was associated with a higher probability of cancer diagnosis in patients with high PSA levels.

\section{Electronic Supplementary Material}

Supplementary materials are available at Cancer Research and Treatment website (https://www.e-crt.org).

\section{Conflicts of Interest}

Conflict of interest relevant to this article was not reported.

\section{Acknowledgments}

Ms. Jung Eun Lee from prostate cancer department contributed to the database management.

\section{References}

1. Constantinou J, Feneley MR. PSA testing: an evolving relationship with prostate cancer screening. Prostate Cancer Prostatic Dis. 2006;9:6-13.

2. Blute ML Jr, Abel EJ, Downs TM, Kelcz F, Jarrard DF. Addressing the need for repeat prostate biopsy: new technology and approaches. Nat Rev Urol. 2015;12:435-44.

3. Mohammed AA. Biomarkers in prostate cancer: new era and prospective. Med Oncol. 2014;31:140.

4. Yli-Hemminki TH, Laurila M, Auvinen A, Maattanen L, Huhtala H, Tammela TL, et al. Histological inflammation and risk of subsequent prostate cancer among men with initially elevated serum prostate-specific antigen (PSA) concentration in the Finnish prostate cancer screening trial. BJU Int. 2013;112: 735-41.

5. De Marzo AM, Platz EA, Sutcliffe S, Xu J, Gronberg H, Drake $\mathrm{CG}$, et al. Inflammation in prostate carcinogenesis. Nat Rev Cancer. 2007;7:256-69.

6. Mahmud S, Franco E, Aprikian A. Prostate cancer and use of nonsteroidal anti-inflammatory drugs: systematic review and meta-analysis. Br J Cancer. 2004;90:93-9.

7. De Marzo AM, Marchi VL, Epstein JI, Nelson WG. Proliferative inflammatory atrophy of the prostate: implications for prostatic carcinogenesis. Am J Pathol. 1999;155:1985-92.

8. Amara S, Tiriveedhi V. Inflammatory role of high salt level in tumor microenvironment (review). Int J Oncol. 2017;50:147781.

9. Liu S, Sun JY, Ren LP, Chen K, Xu B. Propofol attenuates intermittent hypoxia induced up-regulation of proinflammatory cytokines in microglia through inhibiting the activation of NF-Bkappa/p38 MAPK signalling. Folia Neuropathol. 2017;55:124-31.

10. Fassi Fehri L, Mak TN, Laube B, Brinkmann V, Ogilvie LA, Mollenkopf $\mathrm{H}$, et al. Prevalence of Propionibacterium acnes in diseased prostates and its inflammatory and transforming activity on prostate epithelial cells. Int J Med Microbiol. 2011; 301:69-78.

11. Huang TR, Wang GC, Zhang HM, Peng B. Differential research of inflammatory and related mediators in $\mathrm{BPH}$, his- 
tological prostatitis and PCa. Andrologia. [Epub 2018 Feb 14]. https:// doi.org/10.1111/and.12974.

12. Romagny S, Bouaouiche S, Lucchi G, Ducoroy P, Bertoldo JB, Terenzi H, et al. S-Nitrosylation of cIAP1 switches cancer cell fate from TNFalpha/TNFR1-mediated cell survival to cell death. Cancer Res. 2018;78:1948-57.

13. Billis A. Prostatic atrophy: clinicopathological significance. Int Braz J Urol. 2010;36:401-9.

14. Vasavada SR, Dobbs RW, Kajdacsy-Balla AA, Abern MR, Moreira DM. Inflammation on prostate needle biopsy is associated with lower prostate cancer risk: a meta-analysis. J Urol. 2018; 199:1174-81.

15. Busby JE, Evans CP. Determining variables for repeat prostate biopsy. Prostate Cancer Prostatic Dis. 2004;7:93-8.

16. Scattoni V, Maccagnano C, Capitanio U, Gallina A, Briganti A, Montorsi F. Random biopsy: when, how many and where to take the cores? World J Urol. 2014;32:859-69.

17. Ukimura O, Coleman JA, de la Taille A, Emberton M, Epstein JI, Freedland SJ, et al. Contemporary role of systematic prostate biopsies: indications, techniques, and implications for patient care. Eur Urol. 2013;63:214-30.

18. Futterer JJ, Briganti A, De Visschere P, Emberton M, Giannarini G, Kirkham A, et al. Can clinically significant prostate cancer be detected with multiparametric magnetic resonance imaging? A systematic review of the literature. Eur Urol. 2015; 68:1045-53.
19. Mortezavi A, Marzendorfer O, Donati OF, Rizzi G, Rupp NJ, Wettstein MS, et al. Diagnostic accuracy of multiparametric magnetic resonance imaging and fusion guided targeted biopsy evaluated by transperineal template saturation prostate biopsy for the detection and characterization of prostate cancer. J Urol. 2018;200:309-18.

20. Djavan B, Waldert M, Zlotta A, Dobronski P, Seitz C, Remzi $\mathrm{M}$, et al. Safety and morbidity of first and repeat transrectal ultrasound guided prostate needle biopsies: results of a prospective European prostate cancer detection study. J Urol. 2001;166:856-60.

21. Rosenkrantz AB, Taneja SS. Radiologist, be aware: ten pitfalls that confound the interpretation of multiparametric prostate MRI. AJR Am J Roentgenol. 2014;202:109-20.

22. Pal RP, Ahmad R, Trecartan S, Voss J, Ahmed S, Bazo A, et al. A single center evaluation of the diagnostic accuracy of multiparametric magnetic resonance imaging against transperineal prostate mapping biopsy: an analysis of men with benign histology and insignificant cancer following transrectal ultrasound biopsy. J Urol. 2018;200:302-8.

23. Schouten MG, van der Leest M, Pokorny M, Hoogenboom M, Barentsz JO, Thompson LC, et al. Why and where do we miss significant prostate cancer with multi-parametric magnetic resonance imaging followed by magnetic resonance-guided and transrectal ultrasound-guided biopsy in biopsy-naive men? Eur Urol. 2017;71:896-903. 